\title{
Optimal Stochastic Deployment of Heterogeneous Energy Storage in a Residential Multi-Energy Microgrid with Demand-Side Management
}

\author{
Li, Zhengmao; Xu, Yan; Feng, Xue; Wu, Qiuwei
}

Published in:

IEEE Transactions on Industrial Informatics

Link to article, DOI:

10.1109/tii.2020.2971227

Publication date:

2020

Document Version

Peer reviewed version

Link back to DTU Orbit

Citation (APA):

Li, Z., Xu, Y., Feng, X., \& Wu, Q. (2020). Optimal Stochastic Deployment of Heterogeneous Energy Storage in a Residential Multi-Energy Microgrid with Demand-Side Management. IEEE Transactions on Industrial Informatics, 17(2), 991 - 1004. https://doi.org/10.1109/tii.2020.2971227

\section{General rights}

Copyright and moral rights for the publications made accessible in the public portal are retained by the authors and/or other copyright owners and it is a condition of accessing publications that users recognise and abide by the legal requirements associated with these rights.

- Users may download and print one copy of any publication from the public portal for the purpose of private study or research.

- You may not further distribute the material or use it for any profit-making activity or commercial gain

- You may freely distribute the URL identifying the publication in the public portal 


\title{
Optimal Stochastic Deployment of Heterogeneous Energy Storage in a Residential Multi-Energy Microgrid with Demand-Side Management
}

\author{
Zhengmao Li, Student Member, IEEE, Yan Xu, Senior Member, IEEE, Xue Feng, Member, IEEE, and \\ Qiuwei Wu, Senior Member, IEEE
}

\begin{abstract}
The optimal deployment of heterogeneous energy storage (HES), which mainly consists of electrical and thermal energy storage, is essential for increasing the holistic energy utilization efficiency of multi-energy systems. Consequently, this paper proposes a risk-averse method for HES deployment in a residential multi-energy microgrid (RMEMG), considering the diverse uncertainties and multi-energy demand-side management (DSM). Apart from the HES size, location planning, its optimal investment phase is also determined by maximizing the system equivalent daily profit (EDP) and minimizing the risk. To handle the system uncertainties from renewable energy sources (RES), power demands, outdoor temperature, and residential hot water needs, the multi-stage adaptive stochastic optimization (SO) approach is utilized. Then through the constraint linearization and stochastic scenario sampling, the original nonlinear deployment model is converted to a mixed-integer linear programming one and tested on an IEEE 33-bus distribution network based RMEMG. The effectiveness of the proposed method is verified by comparing it with the existing practices. The comparison results indicate that the proposed risk-averse deployment method can effectively increase the system EDP and more immune to the uncertainties. Besides, this method can be practically applied for the emerging RMEMGs, such as smart buildings, intelligent homes, etc., which get long-term DSM contracts.
\end{abstract}

Index Terms-heterogeneous energy storage (HES), risk-averse deployment, residential multi-energy microgrid (RMEMG), multi-energy demand-side management (DSM), multi-stage adaptive stochastic optimization

\section{NOMENCLATURE}

$\begin{array}{ll}\text { Abbreviations } \\ \text { CCHP } & \text { Combined Cooling, Heat, and Power } \\ \text { CVaR } & \text { Conditional value-at-risk } \\ \text { DSM } & \text { Demand-side Management } \\ \text { EB } & \text { Electric Boiler }\end{array}$

Manuscript received March 03, 2019; revised November 10, 2019; accepted January 19,2019 . The work in this paper was supported in part by Ministry of Education (MOE), Republic of Singapore, under grant AcRF TIER 1 2019-T1-001-069 (RG75/19), and in part by National Research Foundation (NRF) of Singapore under project NRF2018 -SR2001-018. Y. Xu's work is supported by Nanyang Assistant Professorship from Nanyang Technological University, Singapore.

Z. Li and Y. Xu are with the School of Electrical and Electronic Engineering, Nanyang Technological University, Singapore. (e-mail: lizh0049@e.ntu.edu.sg, xuyan@ntu.edu.sg)

$\mathrm{X}$. Feng, is with the Engineering Cluster in Singapore Institute of Technology, Singapore. (e-mail: Elsa.Feng@singaporetech.edu.sg).

Q. Wu, is with the Department of Electrical Engineering in Technical University of Denmark, Denmark. (e-mail: qw@elektro.dtu.dk).

\begin{tabular}{|c|c|}
\hline EC & Electric Chiller \\
\hline EDP & Equivalent Daily Profit \\
\hline EES & Electrical Energy Storage \\
\hline GAMS & General Algebraic Modelling System \\
\hline GB & Gas Boiler \\
\hline HES & Heterogeneous Energy Storage \\
\hline MILP & Mixed-integer Linear Programming \\
\hline PBDR & Price-Based Demand Response \\
\hline PV & Photovoltaic Cell \\
\hline RES & Renewable Energy Sources \\
\hline RMEMG & Residential Multi-Energy Microgrid \\
\hline SO & Stochastic Optimization \\
\hline TS & Thermal Storage \\
\hline TST & Thermal Storage Tank \\
\hline VaR & Value-at-risk \\
\hline WT & Wind Turbine \\
\hline \multicolumn{2}{|l|}{ Superscript } \\
\hline$b$ & Index for branches, $\mathrm{b} \in \operatorname{Br}(i, i+1)$ \\
\hline$j$ & Index for the price levels \\
\hline$m$ & $\begin{array}{l}\text { Index for the first year of each investment phase, } \\
m \in p\end{array}$ \\
\hline$p / t / d / i$ & Index for years/dispatch periods/days/buses \\
\hline 4 & Set for $\{p, d, t, i\}$ \\
\hline \multicolumn{2}{|c|}{ Constants and Parameters } \\
\hline$a_{B H}$ & $\begin{array}{l}\text { Correction factor for envelopes like walls, } \\
\text { doors, etc }\end{array}$ \\
\hline$a_{C L}$ & Confidential level \\
\hline$A_{C L}$ & Air volume that infiltrates into the room \\
\hline$C_{A V}$ & Equivalent specific heat of the room \\
\hline$C_{W R} / C_{A i r}$ & Specific heat capacity of water/air \\
\hline$d r$ & Discount rate \\
\hline$I_{L E}$ & Gap length of the door or window crack \\
\hline$K_{H T}$ & Heat transfer coefficient of the room \\
\hline$L_{P L} / L_{Q L}$ & Active/reactive PBDR levels \\
\hline $\mathrm{N}_{\mathrm{F}}$ & The normalized factor for Beta Distribution \\
\hline $\mathrm{N}_{\mathrm{M}}$ & The total number of investment phases \\
\hline$N_{P} / N_{B} / N_{T} / N_{D}$ & $\begin{array}{l}\text { Number of years/buses/dispatch periods/days a } \\
\text { year }\end{array}$ \\
\hline$P_{D G, R E A L}$ & Available energy resource of RES \\
\hline$P_{D L} / H_{H L} / H_{C L}$ & Power/heat/cooling demands \\
\hline$P_{W T} P_{P V}$ & $\begin{array}{l}\text { The power output of wind turbines/photovoltaic } \\
\text { cells }\end{array}$ \\
\hline $\begin{array}{l}P_{D L, P r} / Q_{D L, P r} \\
R / X\end{array}$ & $\begin{array}{l}\text { Active/reactive power demands before the DSM } \\
\text { Branch impedance }\end{array}$ \\
\hline$S_{E S}^{\min } / S_{E S}^{\max }$ & $\begin{array}{l}\text { Minimal/maximal HES capacity for each } \\
\text { bus/group }\end{array}$ \\
\hline
\end{tabular}




\begin{tabular}{|c|c|}
\hline$S_{B H}$ & Area of the building envelope \\
\hline$S_{E S}^{A L L}$ & Maximal energy capacity for the HES system \\
\hline$T_{I W}$ & The temperature of inlet water for water tanks \\
\hline$T_{I N}^{e s t} / T_{S W}^{s e t}$ & Temperature set-point for room/hot water \\
\hline $\mathrm{V}_{0} / \Delta V_{B U S}^{\max }$ & Substation voltage/maximal voltage deviation \\
\hline$V_{R B} / V_{H W}$ & The volume of the room/hot water needed \\
\hline$x_{s}$ & $\begin{array}{l}\text { The generalized symbol for power outputs of } \\
\text { WTs and PVs }\end{array}$ \\
\hline$y_{s}$ & $\begin{array}{l}\text { The generalized symbol for the outdoor } \\
\text { temperature and multi-energy demands }\end{array}$ \\
\hline$\beta_{c x} / \beta_{j x}$ & $\begin{array}{l}\text { Correction factor due to solar irradiation/wind } \\
\text { blow }\end{array}$ \\
\hline$\gamma_{D G}^{\min } / \gamma_{D G}^{\max }$ & Minimal/maximal DG output ratio \\
\hline$\gamma_{E S}^{\max }$ & $\begin{array}{l}\text { The maximal charging/discharging power ratio } \\
\text { of the energy storage to its capacity }\end{array}$ \\
\hline$\gamma_{i t}$ & $\begin{array}{l}\text { The ratio of intrusion loss to basic thermal } \\
\text { transfer loss }\end{array}$ \\
\hline$\gamma_{L L}$ & The leakage loss ratio of the energy storage \\
\hline$\gamma_{S O C}^{\min } / \gamma_{S O C}^{\max }$ & $\begin{array}{l}\text { Minimal/maximal state of charge for energy } \\
\text { storage }\end{array}$ \\
\hline$\Delta t$ & Unit dispatch interval \\
\hline$\Delta T_{V} / \Delta T_{W}$ & $\begin{array}{l}\text { Maximal temperature deviation for room/ hot } \\
\text { water }\end{array}$ \\
\hline$\varepsilon_{\mathrm{SAL}}$ & $\begin{array}{l}\text { The ratio of HES salvage value to its investment } \\
\text { cost }\end{array}$ \\
\hline$\eta_{B C} / \eta_{B D}$ & $\begin{array}{l}\text { Charging/discharging efficiency of battery } \\
\text { storage }\end{array}$ \\
\hline$\eta_{M T}$ & Electrical efficiency of CCHP plants \\
\hline$\eta_{T C} / \eta_{T D}$ & $\begin{array}{l}\text { Charging/ discharging efficiency of thermal } \\
\text { storage }\end{array}$ \\
\hline$\rho_{R K}$ & The weighted risk factor \\
\hline$\theta / \psi$ & Scale parameters for Beta Distribution \\
\hline$\kappa_{M T} / \kappa_{B}$ & $\begin{array}{l}\text { Emission penalty price of CCHP plants/utility } \\
\text { grid }\end{array}$ \\
\hline$\mu / \sigma$ & $\begin{array}{l}\text { Mean/standard deviation for Normal } \\
\text { Distribution }\end{array}$ \\
\hline$\rho_{W R} / \rho_{\text {Air }}$ & Water/air density \\
\hline$\tau_{\mathrm{\top}} / \tau_{B}$ & The decay rate of thermal/battery storage \\
\hline$\chi_{\mathrm{B}} / \chi_{\mathrm{S}}$ & $\begin{array}{l}\text { Power purchasing/selling price between MEMG } \\
\text { and utility grid }\end{array}$ \\
\hline $\begin{array}{l}\chi_{D G}^{M} / \chi_{E S}^{M} \\
\chi_{E S}^{I V}\end{array}$ & $\begin{array}{l}\text { The unit maintenance cost of generators/storage } \\
\text { Unit investment cost of energy storage }\end{array}$ \\
\hline$\chi_{N G}$ & Nature gas price \\
\hline$\chi_{\mathrm{PS}} / \chi_{\mathrm{HS}} / \chi_{\mathrm{CS}}$ & $\begin{array}{l}\text { Power/heat/cooling retail prices to the local } \\
\text { customers }\end{array}$ \\
\hline$\chi_{\mathrm{PS}} / \chi_{\mathrm{HS}} / \chi_{\mathrm{CS}}$ & $\begin{array}{l}\text { Power/heat /cooling retail prices to the local } \\
\text { customers }\end{array}$ \\
\hline $\begin{array}{l}\chi_{S U B} \\
\text { Variables }\end{array}$ & The unit subsidy price for RES \\
\hline$\eta_{V a R}$ & The project VaR \\
\hline$a$ & $\begin{array}{l}\text { Binary indicator for the state of price or load } \\
\text { levels }\end{array}$ \\
\hline$C_{E D P}$ & The project EDP \\
\hline$C_{F C} / C_{T C}$ & Fuel/power transaction cost \\
\hline$C_{I N V} / C_{S A L}$ & Investment cost/salvage value. \\
\hline$C_{M C} / C_{E M}$ & Maintenance/gas emission cost \\
\hline$C_{R E V} / C_{S U B}$ & Revenue from local customers/RES subsidy \\
\hline
\end{tabular}

\begin{tabular}{|c|c|}
\hline$C_{S T} / C_{S D}$ & Start-up/shut-down cost \\
\hline$E_{7} / E_{B}$ & Energy stored in the thermal/battery storage \\
\hline$H_{B H} / H_{A H}$ & Basic/additional thermal transfer loss \\
\hline$H_{C N} / H_{C R}$ & Infiltration/intrusion thermal loss \\
\hline$H_{E B} / H_{E C}$ & Heat/cooling output of electric boilers/chillers. \\
\hline$H_{M H} / H_{M C}$ & Heat/cooling output of CCHP plants \\
\hline$H_{T C} / H_{T D}$ & Charging /discharging power of thermal storage \\
\hline$H_{T L} / H_{N E T}$ & Total space thermal demands/net thermal \\
\hline & The power output of CCHP plants \\
\hline$P_{B C} / P_{B D}$ & Charging/ discharging power of battery storage \\
\hline$P_{B X} / P_{S X}$ & $\begin{array}{l}\text { Purchasing/selling power between MEMG and } \\
\text { utility }\end{array}$ \\
\hline$P_{D G} / P_{E S}$ & Generalized power output for generators/storage \\
\hline$P_{E B} / P_{E C}$ & Power input for electric boilers/chillers. \\
\hline$P_{P F} / Q_{\mathrm{PF}}$ & Active/reactive power flow \\
\hline$P_{R L} / Q_{R L}$ & $\begin{array}{l}\text { Active/reactive power loads after demand } \\
\text { response }\end{array}$ \\
\hline$P_{P F}^{p, d, t, 0, b} / Q_{P F}^{p, d, t, 0, b}$ & Parallel active/ reactive power of branch $b$ \\
\hline$R_{D G, D N} / R_{D G, U P}$ & Maximal ramping down/up limits \\
\hline$S_{E S}$ & The capacity of the energy storage \\
\hline$S_{E S, E q}$ & $\begin{array}{l}\text { The effective capacity of energy storage } \\
\text { considering the leakage loss }\end{array}$ \\
\hline$T_{I N} / T_{O T}$ & Indoor/outdoor temperature \\
\hline$T_{S W}$ & $\begin{array}{l}\text { The temperature of the water inside the water } \\
\text { tank }\end{array}$ \\
\hline$U_{D G}$ & $\begin{array}{l}\text { Binary state to indicate the on-off status of } \\
\text { generators }\end{array}$ \\
\hline$U_{E S}$ & $\begin{array}{l}\text { Binary state to denote investment decisions for } \\
\text { HES }\end{array}$ \\
\hline$U_{E S C} / U_{E S D}$ & Binary charging/discharging states of HES \\
\hline & Bus voltage \\
\hline
\end{tabular}

\section{INTRODUCTION}

WERGY storage is to capture the energy produced at one - time for later use [1]. Its optimal deployment in the energy systems is essential for providing numerical benefits, such as tackling the intermittent power outputs from RES, achieving higher energy utilization flexibility, shaving peak energy demands, improving the voltage profile, etc. [2]-[3].

For the existing research on the deployment of energy storage, currently, it focuses mainly on electrical energy storage (EES). Ref. [4] formulates an optimal placement model for the EES in a power network to shift loads and minimize the generation cost. In [5], a cost-effective method for EES sizing in the distribution system is proposed. This method decides the optimal EES capacity regarding its operating cost under different RES penetration levels. Ref. [6] presents a model for integrating EES in a microgrid at the minimum cost, to analyze its potential advantages with different conceivable regulatory services. Ref. [7] determines the optimal EES size in the RES-integrated power system to get the minimal system operating cost. However, in the above research, the EES only facilitates RES integration without considering a system-wide operation. Hence, their deployment solutions may not be optimal from the system perspective. Therefore, ref. [3] studies the EES deployment in a microgrid based on cost-benefit analysis. The time series and neural network techniques are 
used to forecast the wind speed and solar radiations, respectively. In [8], to determine the optimal EES size and location, the probabilistic load model is adopted to incorporate more load scenarios. The above research has utilized the deterministic optimization method by assuming that there is perfect information about the RES generation and end-user energy consumption. Nonetheless, the RES and energy consumption are intrinsically uncertain, which makes the solutions from the deterministic method unreliable.

In the literature, to deal with system uncertainties in the deployment of energy storage, various approaches are employed. In [9], the optimal EES deployment is obtained by the deterministic optimization tool with the uncertainties addressed by the model predictive control method. Ref. [10] studies the optimal EES sizing problem to minimize the annual cost by a bi-level programming method. The uncertainties are managed by the system reserve. Though the uncertainties are considered, both works still use deterministic models, which fail to provide a reliable or robust deployment plan. Regarding this, the stochastic and rubout optimization approaches have been employed. Authors in [11] propose a stochastic optimization (SO) method for EES sizing in a wind-dieselbased isolated grid. In [12], the optimal EES sizing problem in microgrids with highly penetrated wind energy is solved by the unit-commitment-based robust optimization method. Authors in [13] investigate an efficient cost-reliability optimization method to obtain the optimal EES location and size in a microgrid considering the DSM for electricity. To handle the uncertainties from wind turbines (WTs), the SO method is utilized. Ref. [14] introduces the SO approach for EES deployment in remote systems, to reduce the system operating cost. Authors in [15] propose a joint robust capacity optimization method for EES and distributed generators to save the operating cost under the fluctuating RES outputs. For the long-term planning research, the worst-case-oriented robust optimization method tends to be too conservative compared with the stochastic one, and highly depends on the predefined uncertainty budgets. As for the stochastic approach, currently, the majority are risk-neutral [13], [14], however, neglecting the risk will result in a threat to the reliability of microgrids. In this regard, the project managers would be willing to choose a solution with higher operational costs but lower risk [16].

Besides, most of the existing research work focuses only on the deployment of EES in the power system. Nonetheless, recent years have seen a dramatic development of multi-energy systems, which integrate the increasing power, heat, and cooling energy carriers [17]. At the distribution network level, multi-energy systems are usually realized in the form of RMEMGs, such as smart buildings, intelligent homes, etc [18], which require plenty of thermal demands. In this regard, to effectively enhance the holistic energy efficiency and operation flexibility, the EES deployment is shifted to HES deployment in the RMEMGs, with the uncertainties from only the power system to multi-energy systems, such as the outdoor temperature or thermal demands [19]. However, in the literature, very limited research work has been done in this area. Ref. [20] plans the HES size aiming to minimize the project cost and gas emissions by a two-stage solution method. The first stage uses the genetic algorithm to solve the planning problem and the second stage applies the MILP solvers to get the operating solution. In [21], both the system structure and HES capacity configuration are studied in a planning framework of the smart microgrid. Then, a MILP based planning model was proposed to minimize the total operating cost. The study in [22] proposes a novel optimal design model for a microgrid that involves HES. To reduce the computational burden, the model uses the typical design days to represent the yearly horizon. Though the above research investigates the HES deployment, the network constraints and the effects of multi-energy uncertainty sources are totally ignored. In this regard, the system reliability cannot be guaranteed, and their solutions are not practical for the real application.

Furthermore, as the DSM is a promising and effective solution to support the system operation as well as save energy bills for residents, currently, there is a widespread installation of the smart meters in residential buildings with a long-term DSM contract [18]. Compared with the electricity, thermal energy (heat or cooling) gets higher inertia, as residents can be comfortable within a tolerable temperature range [23]. In this sense, though the DSM for electricity is widely studied in the areas such as power grid transmission planning [24], robust operation [25], low-carbon sustainability [26], etc., its coordination with the thermal energy is rarely studied especially for the HES deployment of RMEMGs.

Last but not least, most of the existing deployment works, either for EES or HES only consider the one-off installation at the beginning of the project as they ignore the increasing load growth. Thus, their deployment decisions would be probably far from enough, given the annual load growth in reality [27]. In this regard, the multi-phase deployment, in which the HES can be invested at different years of the project, is critical for the more comprehensive and flexible planning research.

To fill the research gap, in this paper, an optimal risk-averse stochastic HES deployment method is proposed in a grid-tied RMEMG under diverse uncertainties. Compared with the existing research works, the contributions in this paper are summarized as follows.

1) A new risk-averse HES deployment model in the grid-tied RMEMGs is proposed. Instead of focusing merely on EES, its integration with the thermal storage (TS) and different energy properties are also investigated.

2) For more comprehensive and flexible HES deployment work, in addition to the traditional size and location planning, the investment phase selection is also considered, given in reality that there is annual load growth.

3) Diverse uncertainties not only from RES and power loads of the traditional power system but from the outdoor temperature and hot water needs of the thermal system are also taken into full account. Then, a scenario-based multi-stage adaptive SO approach is proposed to tackle all the uncertainties.

4) The DSM for multiple energies is incorporated in the HES deployment, given its advantage in flexibilizing the system operation and reducing the energy bills. Especially for the 
thermal system, the DSM in terms of specific thermal modeling for the local residential buildings is built with thermal dynamics and thermal inertia combined.

For the practical implications of this work, with the increasing number of green buildings and smart homes, that are partially powered by the RES and have signed long-term multi-energy DSM contracts, the proposed method can be directly applied to the deploy their HES system.

The rest of this paper is organized as follows. Section II describes the basic structure of the RMEMG with the specific modeling of all its units. Section III introduces the proposed risk-averse HES deployment model and its scenario-based multi-stage adaptive SO form. Section IV presents a detailed case study and simulation results of the HES deployment. At last, Section V concludes the whole paper.

\section{Residential Multi-ENERGy Microgrid Modelling}

\section{A. Residential Multi-energy Microgrid Structure}

The structure of a typical RMEMG is shown in Fig.1. Its main components are the distributed generators, HES system, and multi-energy demands. The RMEMG is grid-tied, which means its power can be transacted with the main grid [18]. Further, the residents are assumed to have signed a long-term contract with the RMEMG to save their energy bills.

The inner distributed generators can be classified as [27]:

1) dispatchable generators, which are in small sizes, flexible control, and high ramp rates: electric boilers (EBs), combined cooling, heat, and power (CCHP) plants with the main generator as micro-turbines, and electric chillers (ECs);

2) non-dispatchable generators, whose energy outputs are highly uncertain and volatile: WTs and photovoltaic cells (PVs).

For the HES system, it is mainly composed of the power energy type, i.e., battery storage, and thermal energy type, i.e., thermal storage tank.

All the units are coordinated to satisfy the multi-energy demands as well as maintain the system reliability and economy [19]. The RES generation, power demands, outdoor temperature, residential hot water needs, and demand response pattern data can all be collected by the smart meters equipped in each building. The collected data is then applied in the computation procedure as optimization parameters.

The detailed computation procedures are described in Section. III and IV. The deployment decisions can be obtained from those procedures.



Fig.1 Typical Structure of a RMEMG.

\section{B. Battery Storage (BS)}

The BS is a kind of prevailing EES widely used in power system planning and operation. Compared with other types of EES such as the pumped storage and compressed air energy storage with capacities up to $100 \mathrm{MW}$, its power density range starts from a few $\mathrm{kW}$ to several MW. This makes BS more suitable for the application in microgrid or community level [2], [6].

The relationship between energy and power of BS can be denoted as:

$$
E_{B}^{t}=E_{B}^{t-1}\left(1-\tau_{B}\right)+P_{B C}^{t} \eta_{B C} \Delta t-P_{B D}^{t} \eta_{B D} \Delta t
$$

\section{Thermal Storage Tank (TST)}

The typical TST model is shown in Fig. 2. Its walls are composed of stainless steel, concrete, and heat insulation materials from inside to outside. The tank is well insulated for high operating efficiency [17]. The temperature in the tank can be roughly stratified into the hot water layer (Temperature: $T_{h}$, Volume: $V_{h}$ ) and cooling water layer (Temperature: $T_{c}$, Volume: $\mathrm{V}_{\mathrm{c}}$ ). In both layers, the temperature is the same as the corresponding water networks and remains constant. The TST absorbs or releases thermal energy by controlling the volume of hot or cold water. When it produces heat, the temperature of the supply water becomes higher, in this light, the TST is called a heat storage tank; while for the cooling supply, the TST should be called a cooling storage tank with a higher temperature of return water. 




Fig.2 Structure of the TST. SI, SO: inlet and outlet switch for supply water; RI, $\mathrm{RO}$ inlet and outlet switch for return water.

The absorbing and releasing power of TST can be expressed as follows:

$$
\begin{aligned}
& H_{T C}^{t}=C_{W R} \cdot \rho_{W R} \cdot V_{H}^{t} \cdot\left(T_{H}-T_{C}\right) \\
& H_{T D}^{t}=-C_{W R} \cdot \rho_{W R} \cdot V_{C}^{t} \cdot\left(T_{C}-T_{H}\right)
\end{aligned}
$$

The relationship between its energy and power is:

$$
E_{T}^{t}=E_{T}^{t-1}\left(1-\tau_{T}\right)+H_{T C}^{t} \eta_{T C} \Delta t-H_{T D}^{t} \eta_{T D} \Delta t
$$

\section{Price-based Demand Response (PBDR) for Electricity}

The PBDR is adopted for electricity DSM in the RMEMG. The reason lies in twofold: first, it allows customers to manage demand freely without partial loads being controlled directly by the system operator. In the PBDR, electricity prices of the following day are designed and delivered to the customers a day ahead, then the customers adjust their demands based on the prices. When the prices are high, the customers would reduce their demands to save bills. When they are low, customers would be incented to increase their demands; second, it contributes to the system reliability enhancement when there is a large penetration level of fluctuating RES [25].

Currently, the PBDR has been widely applied in the power system planning, power market regulation, and economic dispatch, etc. In the PBDR model, the relationship between the electricity price and demands can be expressed as follows:

$$
P_{D L}^{t}=A \cdot\left(\chi_{P S}\right)^{\varepsilon}
$$

In Eq.(5), $\varepsilon$ is the price elasticity of power demands, representing the sensitivity of customer demand to the price of electricity; A is a constant. The specific calculation methods of $\varepsilon$ and A are given by [28].

\section{E. Thermal Loads Modelling with Thermal Inertia}

To comprehensively model the thermal energy in a typical residential building, its typical structure is demonstrated in Fig.3 [29].



Fig.3 Typical structure of a room in a residential building.

For the residents, their thermal demands mainly come from the following two parts:

1) space thermal demands, i.e., space heating (usually in winter) and cooling (usually in summer) [19].

2 ) residential hot water needs (usually in spring, autumn, and winter) [30].

All the above thermal demands can be modeled considering the thermal dynamics.

\section{1) Thermal Demand Classification}

For space thermal demands, the thermal generation should compensate for any thermal loss to maintain the suitable room temperature. The total thermal demands are calculated as [31]:

$$
H_{T L}^{t}=H_{B H}^{t}+H_{A H}^{t}+H_{C N}^{t}+H_{C R}^{t}
$$

The basic thermal transfer loss mainly includes the heat transfer from the building envelope such as doors, windows, walls, floors, and so on, which can be denoted as Eq.(7):

$$
H_{B H}^{t}=\alpha_{B H} K_{H T} S_{B H}\left(T_{I N}^{t}-T_{O T}^{t}\right)
$$

The additional thermal loss considers the loss brought by the wind blow, solar irradiation, and height of the building. It is calculated as:

$$
H_{A H}^{t}=H_{B H}^{t} \cdot\left(\beta_{c x}+\beta_{\mathrm{jx}}\right)
$$

The infiltration thermal loss denotes the thermal demands to heat the cold air (mainly in winter) or cool the hot air (mainly in summer) from outside to inside via the door or window crack. It is formulated as:

$$
H_{C N}^{t}=0.364 \cdot I_{L E} A_{C L}\left(T_{I N}^{t}-T_{O T}^{t}\right)
$$

The intrusion thermal loss describes the thermal demands that cool the hot air (mainly in summer) or heat the cold air (mainly in winter) from the casual opening of the doors or windows. It can be demonstrated as:

$$
H_{C R}^{t}=H_{B H}^{t} \gamma_{i t}
$$

All the above thermal loss is related to the difference between inside and outside temperature, then the total thermal demands can be rewritten in a general form as:

$$
H_{T L}^{t}=C_{A V}\left(T_{I N}^{t}-T_{O T}^{t}\right)
$$

\section{2) Dynamic Thermal Demand Modelling}

According to the first law of thermodynamics, the thermal demands in Eqs. (6)-(11) can be modeled as a dynamic model with the temperature change as:

$$
C_{A i r} \rho_{A i r} V_{R B} d T_{I N}^{t} / d t=H_{N E T}^{t}
$$

Due to the thermal inertia, the indoor temperature would vary slowly. Its variations during each short-term period, e.g., 1 hour can then be neglected [19], [29]. In this regard, the thermal 
dynamic model in Eq. (12) can be converted into an hourly state model as:

$$
T_{I N}^{t}=H_{N E T}^{t} \Delta t /\left(C_{A i r} \rho_{A i r} V_{R B}\right)+T_{I N}^{t-1}
$$

Note that human activities, power appliances, etc., may also impact the space thermal demands, but as the impact is little, they are ignored in this work [2], [31].

3) Hot water demands

The hot water is mainly used for the showering or washing needs of the residents. The corresponding thermal demands for hot water can be calculated as [29]:

$$
H_{H W}^{t}=C_{W R} \rho_{W R} V_{H W}^{t}\left(T_{S W}^{t}-T_{I W}^{t}\right)
$$

\section{F. The DSM for Thermal Energy}

Apart from the slow variation rate of thermal energy during short periods, the fact that residents can be comfortable within a certain indoor or hot-water temperature range constitutes another aspect of the thermal inertia [30]. This part is regarded as the DSM for thermal energy. It can be modeled as Eqs.(15) and (16).

$$
\begin{aligned}
T_{I N}^{s e t}-\Delta T_{V} & \leq T_{I N}^{\Lambda} \leq T_{I N}^{s e t}+\Delta T_{V} \\
T_{S W}^{s e t}+\Delta T_{W} & \leq T_{S W}^{\Lambda} \leq T_{S W}^{s e t}+\Delta T_{W}
\end{aligned}
$$

\section{MATHEMATIC MODELling For HES Deployment}

\section{A. Multi-stage HES Deployment Framework}

In this study, the HES deployment can be decomposed into two main stages as Fig. 4 with respective decisions:



Fig. 4 Multi-stage HES deployment framework.

1) Investment stage: decisions at this stage are related to the investment aspects, namely the HES location, size, and investment phase. As shown in Fig.4, 3 years is taken as a unit investment phase, and after every 3 years, there can be new HES investment decisions.

2) Operation stage: decisions at this stage include the optimal dispatch of the HES system, distributed generators and DSM scheme. The diverse uncertainties are handled via a further two-substage coordinated operation method. The first substage is the day-ahead dispatch stage; the second substage is the intraday online dispatch stage. The details for the two substages are given in Section. IV.

\section{B. Mathematical Formulation}

In this study, it is assumed there is a steady growth rate for the multi-energy demands every year. To evaluate the effectiveness of the method, the maximization of "mean-risk", which is equal to the subtraction of EDP and risk cost is set as the objective. In finance, the EDP is defined as the profit per day of owning and operating an asset over its entire lifespan. It is calculated by dividing the net present value of a project by the present value of the daily factor. The risk cost can be measured by the conditional value-at-risk (CVaR) [16], [32]. Then, the risk-averse HES deployment model can be formulated as:

1) Objective:

$$
\begin{aligned}
& \max _{z \in Z, x \in X, \eta_{\text {VaR }} \in \Re}\left[C_{E D P}-\rho_{R K} C \operatorname{VaR}_{\alpha}\left(C_{E D P}\right)\right] \\
& \text { s.t., } C_{E D P}=\frac{1}{365 \times N_{P}} \cdot \frac{d r(1+d r)^{N_{P}}}{(1+d r)^{N_{p}}-1} \cdot[\underbrace{-F(z)}_{\text {Investment Stage }}+\underbrace{G(x)}_{\text {Operation Stage }}](18) \\
& C \operatorname{VaR}_{\alpha}\left(C_{E D P}\right)=\eta_{V a R}+\frac{1}{1-\alpha_{C L}} \mathrm{E}\left[\max \left(C_{E D P}-\eta_{V a R}, 0\right)\right] \\
& F(\mathrm{z})=\sum_{p \in N_{P}} \frac{C_{I N V}^{p}}{(1+d r)^{p-1}}-\frac{C_{S A L}^{N_{P}}}{(1+d r)^{N_{P}-1}} \\
& G(x)=\sum_{p \in N_{p}} \frac{\left(C_{R E V}^{p}+C_{S U B}^{p}-C_{F C}^{p}-C_{T C}^{p}-C_{M C}^{p}-C_{E M}^{p}-C_{S T}^{p}-C_{S D}^{p}\right)}{(1+d r)^{p}} \\
& C_{S A L}^{N_{P}}=\sum_{p \in N_{P}} \varepsilon_{S A L}^{p} C_{I N V}^{p} \\
& C_{I N V}^{p}=\sum_{i \in N_{B}} \chi_{E S, I N V}\left(S_{E S}^{p, i}-S_{E S}^{p-1, i}\right) \\
& C_{R E V}^{p}=\sum_{d \in N_{D}} \sum_{t \in N_{T}} \sum_{i \in N_{B}}\left(\chi_{P A}^{\Lambda} P_{D L}^{\Lambda}+\chi_{H S} H_{H L}^{\Lambda}+\chi_{C S} H_{C L}^{\Lambda}\right) \\
& C_{S U B}^{p}=\sum_{d \in N_{D}} \sum_{t \in N_{T}} \sum_{i \in N_{B}} \chi_{S U B}\left(P_{W T}^{\Lambda}+P_{P V}^{\Lambda}\right) \\
& C_{F C}^{p}=\sum_{d \in N_{D}} \sum_{t \in N_{T}} \sum_{i \in N_{B}}\left(\chi_{N G} P_{M T}^{\Lambda} / \eta_{M T}\right) \\
& C_{T C}^{p}=\sum_{d \in N_{D}} \sum_{t \in N_{T}} \sum_{i \in N_{B}}\left(\chi_{B} P_{B X}^{\Lambda}-\chi_{S} P_{S X}^{\Lambda}\right) \\
& C_{M C}^{p}=\sum_{i \in N_{B}} \sum_{d \in N_{D}} \sum_{t \in N_{T}} \chi_{D G}^{M} P_{D G}^{\Lambda}+\chi_{E S, M} P_{E S}^{\Lambda} \\
& C_{E M}^{p}=\sum_{d \in N_{D}} \sum_{t \in N_{T}} \sum_{i \in N_{B}} \kappa_{M T} P_{M T}^{\Lambda}+\kappa_{B} P_{B X}^{\Lambda} \\
& C_{S T}^{p}=\sum_{d \in N_{d}} \sum_{t \in N_{T}} \sum_{i \in N_{B}} \max \left\{0, U_{D G}^{\Lambda}-U_{D G}^{p, d, t-1, i}\right\} C_{D G}^{U} \\
& C_{S D}^{p}=\sum_{d \in N_{d}} \sum_{t \in N_{T}} \sum_{i \in N_{B}} \max \left\{0, U_{D G}^{p, d, t-1, i}-U_{D G}^{\Lambda}\right\} C_{D G}^{D}
\end{aligned}
$$

2) Constraints:

$$
\begin{gathered}
\text { Eqs.(1)-(16) } \\
S_{E S}^{p, i} \geq S_{E S}^{p-1, i} \\
U_{E S}^{p, i} \cdot S_{E S}^{m i n} \leq S_{E S}^{p, i} \leq U_{E S}^{p, i} \cdot S_{E S}^{m a x} \\
\sum_{i \in N_{B}} S_{E S}^{p, i} \leq S_{E S}^{A L L} \\
S_{E S, E q}^{p, i}=\sum_{m \in N_{M}}\left(S_{E S}^{m, i}-S_{E S}^{m-1, i}\right)\left(1-\gamma_{L L}\right)^{p-m}, p \geq m \\
0 \leq P_{E S C}^{\Lambda} \leq \gamma_{E S}^{m a x} U_{E S C}^{\Lambda} S_{E S, E q}^{p, i} \\
0 \leq P_{E S D}^{\Lambda} \leq \gamma_{E S}^{\max } U_{E S D}^{\Lambda} S_{E S, E q}^{p, i} \\
U_{E S C}^{\Lambda}+U_{E S D}^{\Lambda} \leq 1 \\
\sum_{i \notin N_{B}} U_{E S}^{p, i}=0 \\
\gamma_{S O C}^{\min } S_{E S, E q}^{p, i} \leq E_{E S}^{\Lambda} \leq \gamma_{S O C}^{m a x} S_{E S, E q}^{p, i} \\
E_{E S}^{p, d, 0, i}=E_{E S}^{p, d, N_{T}, i} \\
\gamma_{D G}^{m i n} S_{D G}^{p, i} \leq P_{D G}^{\Lambda} \leq \gamma_{D G}^{\max } S_{D G}^{p, i}
\end{gathered}
$$




$$
\begin{gathered}
P_{D G}^{\Lambda} \leq P_{D G, R E A L}^{\Lambda} \\
R_{D G, D N}^{\Lambda} \Delta t \leq P_{D G}^{\Lambda}-P_{D G}^{p, d, t-1, i} \leq R_{D G, U P}^{\Lambda} \Delta t \\
P_{P F}^{p, d . t, b+1}=P_{P F}^{p, d, t, b}-P_{P F}^{p, d, t, 0, b+1}-P_{D L}^{\Lambda}+P_{M T}^{\Lambda}+P_{E P}^{\Lambda}- \\
P_{C P}^{\Lambda}+P_{W T}^{\Lambda}+P_{P V}^{\Lambda}-P_{E B}^{\Lambda}-P_{E C}^{\Lambda} \\
Q_{P F}^{p, d, t, b+1}=Q_{P F}^{p, d, t, b}-Q_{P F}^{p, d, t, 0, b+1}-Q_{D L}^{p, d, t, i} \\
V_{B U S}^{p, d, t, i+1}=V_{B U S}^{p, d, t, b}-\left(R^{b} P_{P F}^{p, d, t, b}+X^{b} Q_{P F}^{p, d, t, b}\right) / V_{0} \\
1-\Delta V_{B U S}^{\max } \leq V_{B, S}^{p, d, t, i} \leq 1+\Delta V_{B U S}^{\max } \\
H_{T L}^{\Lambda}+H_{W R}^{\Lambda}=H_{M H}^{\Lambda}+H_{E B}^{\Lambda}+H_{M C}^{\Lambda}+H_{E C}^{\Lambda}+H_{T D}^{\Lambda}-H_{T C}^{\Lambda}
\end{gathered}
$$

Eq.(17) denotes the objective of the risk-averse HES deployment model [32]. Eq.(18) is the mathematical formulation of the system EDP in which $\mathrm{z}$ and $\mathrm{x}$ denote the set of investment- and operation-stage decisions; Eq. (19) denotes the system risk cost in terms of CVaR. F(z) in Eq. (20) and $\mathrm{G}(z)$ in Eq.(21) denote the cost from the investment- and operationstage, respectively; Eq.(22) denotes the salvage value of the HES system; Eq.(23) presents the investment cost of the HES system; Eqs.(24) is the revenue from selling power and thermal energy to local residents; Eqs.(25) is the subsidy from RES utilization; Eqs.(26)-(29) denote the CCHP fuel cost, power transaction cost, maintenance cost from all the units as well as emission cost from CCHP plants and utility grid; Eqs.(30)-(31) are the start-up and shut-down cost for dispatchable generators; Eq.(33) means that the size of the HES system in the present year should be no less than that of the last year. It is assumed that HES can be available in all variable sizes [19]; Eqs.(34) denotes HES size limits for each bus or thermal group. Eqs. (35) describes the HES size limits for the RMEMG; Eqs.(36) is the effective capacity of the energy storage considering the leakage loss. It can be seen that the capacity of the energy storage is decreasing gradually after the investment [33]; Eqs.(37)-(42) denote all the HES operating constraints (subscript ES denotes the generalized abbreviation for BS and TST); Eqs.(37) and (38) mean that the charging (absorbing) or discharging (releasing) power of HES should be within the capacity limits; Eq.(39) shows that HES cannot charge (absorb) and discharge (release) at the same time; Eq.(40) denotes that the HES can only be installed at the candidate buses or thermal groups; Eq.(42) means that the HES starting energy is equal to its ending energy for the same scheduling flexibility of each dispatch horizon [19]; Eq.(43) denotes that the power output of each generator should be within their capacity limits (subscript DG is the generalized abbreviation for all the generators), while Eq.(44) shows that the RES power output should be less than available sources; Eq.(45) presents the ramp up and down rate limits for the controllable generators; Eqs.(46)-(48) are the distribution network's power flow, which is modeled based on the linearized Dist-Flow equations in [2], [19]; Eq.(49) denotes that bus voltage should be maintained in a secure range; Eq.(50) denotes that thermal generation should be equal to its consumption [17], [27].

Note that, in the short-term operation, there would be degradation cost for energy storage [34], [35], however, it is ignored in this long-term deployment work as we aim to find the rough planning decisions for the HES [12], [20], [22].
In the HES deployment model, there are a number of nonlinear items such as Eqs.(5), (19),(30)-(31) and (37)-(38). To reduce the computation burdens as well as improve the solution quality, those nonlinear items are linearized in Appendix A. After linearization, the proposed HES deployment model becomes a MILP problem.

\section{Solution Method}

\section{A. Typical Seasonal Day Selection}

Given that there are 365 days in a year, the HES deployment model is in excessively high dimension and thus computationally demanding. To prune the model's size, a set of typical seasonal days is chosen to represent the yearly horizon. For example, the EDP can be converted as:

$$
C_{E D P}=\frac{1}{365 \times N_{P}} \cdot \frac{d r(1+d r)^{N_{P}}}{(1+d r)^{N_{p}}-1} \cdot[\underbrace{-F(z)}_{\text {Investment Stage }}+\underbrace{\sum_{c \in N_{C}} N_{D}^{c} \cdot G(x \mid c)}_{\text {Operation Stage }}]
$$

where $N_{D}^{c}$ is the number of days in season $c ; N_{C}$ is the number seasons; $G(x \mid c)$ is the objective of operation-stage in season $c$.

\section{B. Scenario-based Stochastic Operation Model}

To handle the diverse uncertainties, a two-substage adaptive SO method is proposed as Fig.5.



Fig. 5 Two-substage coordinated operation framework.

In Fig.5, the operation-stage is further decomposed into the day-ahead and intraday online dispatch substages. Given that the HES system and PBDR are dynamic and sensitive to the holistic price information, their 24-hour dispatch decisions (one hour as the unit dispatch interval [2]) are made in the day-ahead dispatch substage. At this substage, the diverse uncertainties are not revealed in real-time. In the intraday online dispatch substage, where the diverse uncertainties are realized, all the dispatchable generators that have faster response speeds are coordinated (15 minutes as the unit dispatch interval) to maintain the real-time balance of multi-energy generation and consumption.

\section{1) Uncertainty Modelling}

For the energy outputs of WTs and PVs, their stochastic variations or forecasting errors usually follow the Beta distribution as:

$$
f_{x_{s}}=\left(x_{s}\right)^{\theta-1} \cdot\left(1-x_{s}\right)^{\psi-1} \cdot N_{F}
$$

As for the power demands, outdoor temperature and residential hot water needs, Normal distribution can be employed to represent their forecasting errors [19]:

$$
f_{y_{s}}=1 /(\sqrt{2 \pi} \sigma) \cdot \exp \left[-\left(y_{s}-\mu\right)^{2} /\left(2 \sigma^{2}\right)\right]
$$

\section{2) Scenario Generation and Reduction}

Based on the probability distributions of diverse uncertain variables in Eqs.(52)-(53), Latin hypercube sampling method can be used to generate a large number of near-random samples 
of parameter values for the stochastic model [27].

However, the generated number of scenarios would lead to a significant computational burden. In this regard, the simultaneous backward reduction method is implemented to choose a much smaller but rather representative set of scenarios for the practical applications.

The scenario generation and reduction method employed in this work have already been applied in the scenario processing. The specific details can be referred to [13],[19], [27].

After the scenario generation and reduction, the stochastic model is modeled as a MILP problem as Eqs.(54)-(55):

$$
\begin{array}{ll}
\underset{x}{\operatorname{Min}} G(x) & =\operatorname{Min}_{w, y_{1}, y_{2} \ldots, y_{q}}\left[S(w)+\sum_{q \in N_{Q}} c_{q} L\left(y_{q}\right)\right] \\
\text { s.t. } & w \in C S_{w} \mid z \\
& y_{q} \in C L\left(w, \omega_{q}\right), \forall q
\end{array}
$$

where $q$ is the index of the forecasted scenarios; $\mathrm{N}_{\mathrm{Q}}$ is the total number of scenarios; $\mathrm{c}_{\mathrm{q}}$ is the scenario possibility; $\mathrm{S}(w)$ is objective of investment stage and day-ahead dispatch stage; $\mathrm{w}$ denotes the decision variables for size, location, investment phase and corresponding dispatch of HES system as well as PBDR scheme; $\mathrm{CS}_{\mathrm{w}}$ is constraints associated with $\mathrm{w} ; \mathrm{E}\left[\mathrm{L}\left(\mathrm{y}_{\mathrm{q}}\right)\right]$ is the expectation of cost from the dispatchable generators and power transaction between the RMEMG and utility grid; yq denotes the corresponding decision variables for power flow, dispatchable generators, and temperature for the rooms and hot water; $\omega_{\mathrm{q}}$ is stochastic variation of system uncertain sources; $\mathrm{CL}\left({ }_{\mathrm{w}}, \omega_{\mathrm{q}}\right)$ is the constraints associated with $y_{q}$.

\section{Simulation Results}

\section{A. Test System}

The test grid-tied RMEMG is built with reference to the real conditions in Tianjin ( $\left.38^{\circ} \mathrm{N}, 117^{\circ} \mathrm{E}\right)$, China, as shown in Fig.6. Its lifetime is 9 years with 3 years as the unit investment phase. The multiple energies are served mainly for the neighboring aggregated residential buildings, which are in three regions. The heat networks among different thermal groups are not considered in this work since the long-distance thermal transmission will result in substantial thermal loss [36]. The predefined configuration of all the generators is shown in Table. I, while the substation capacity is set as 1.5 times of the peak power in RMEMG [2] to guarantee that there is enough power supply when the faults occur inside. The technical details for the HES system are listed in Table. II. The studied RMEMG in Fig. 6 is built based on the IEEE 33-bus standard test system in [37] with all the system data.

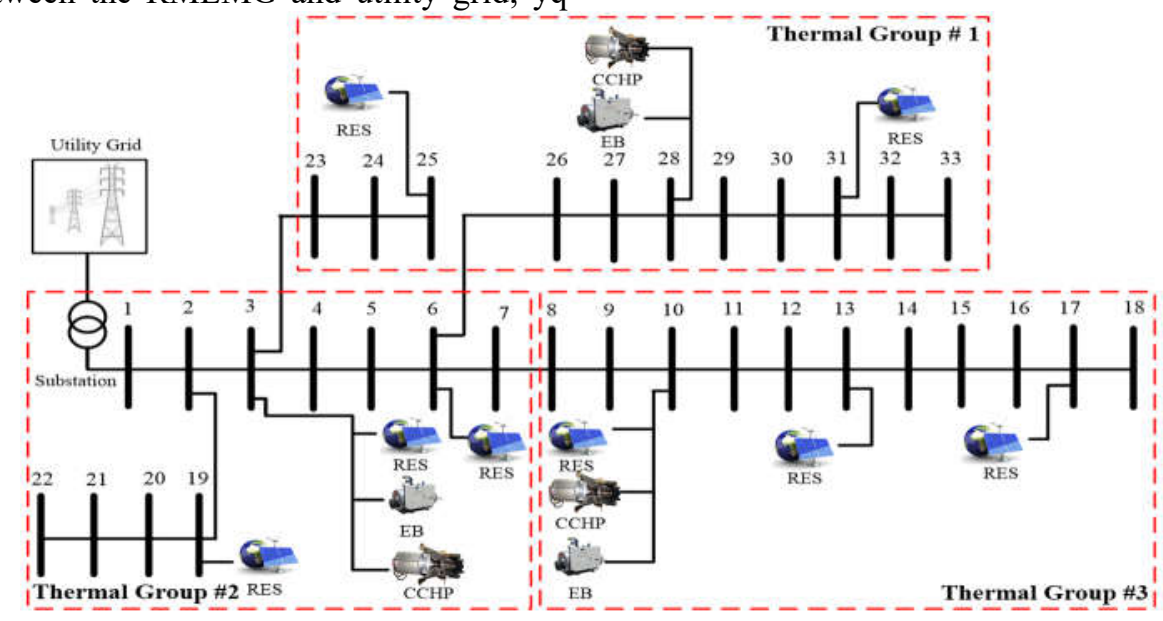

Fig. 6 The IEEE 33-bus radial distribution system based RMEMG.

Three typical days from the transition seasons (spring and autumn), summer and winter are used to represent the year. Based on the data of the year 2018 (Jan.-Dec.), Fig. 7 shows the forecasting value of all the uncertain sources in the year 2019.



(a) Transition seasons



(c) Winter



(b) Summer



(d) Temperature and water needs
Fig. 7 Forecasted RES output, outdoor temperature, and multi-energy demands. TABLE I

CONFIGURATION OF DistRIBUTED GENERATORS (KW)

\begin{tabular}{cccccc}
\hline \hline Node & WT & PV & CCHP & EB & EC \\
\hline 3 & 150 & 40 & 1200 & 650 & 500 \\
6 & 187.5 & 80 & 0 & 0 & 0 \\
10 & 187.5 & 64 & 1000 & 500 & 450 \\
13 & 112.5 & 64 & 0 & 0 & 0 \\
17 & 187.5 & 96 & 0 & 0 & 0 \\
19 & 150 & 128 & 0 & 0 & 0 \\
25 & 187.5 & 144 & 0 & 0 & 0 \\
28 & 0 & 0 & 800 & 500 & 350 \\
31 & 187.5 & 80 & 0 & 0 & 0 \\
\hline \hline
\end{tabular}

The system energy tariffs are shown in Table. III, which are extracted from [17], [19],[20], [38]. For the thermal and power energy supplied to residents, the fixed pricing scheme is applied [2]. According to the electricity price and usage data in [39], the price elasticity of electricity demands is estimated as -0.75 .

TABLE II

TECHNICAL DeTAILS FOR THE HES SySTEM [19],[20] 


\begin{tabular}{cccc}
\hline \hline Energy Storage & $\chi E S, I N V$ & $\chi_{E S, M}$ & $S_{E S, M I N} / S_{E S, M A X}$ \\
\hline Battery storage & $100(\$ / \mathrm{kWh})$ & $0.03(\$ / \mathrm{kWh})$ & $0 / 1500(\mathrm{kWh})$ \\
Heat storage tank & $36.7(\$ / \mathrm{kWh})$ & $0.025(\$ / \mathrm{kWh})$ & $0 / 1800(\mathrm{kWh})$ \\
Cooling storage tank & $36.7(\$ / \mathrm{kWh})$ & $0.025(\$ / \mathrm{kWh})$ & $0 / 1800(\mathrm{kWh})$ \\
\hline \hline
\end{tabular}

In the RMEMG, there are 16 buildings oriented South with 12 floors in each building; On each floor, there are two identical apartments, each with a floor area of $150\left(15^{*} 10\right) \mathrm{m}^{2}$; The area of north/south and east/west facing walls are $42(15 * 2.8) \mathrm{m}^{2}$ and $28(10 * 2.8) \mathrm{m}^{2}$ respectively; The south and north walls are equipped with the double-glazed windows to the outside environment with areas of $10.5(3.75 * 0.7) \mathrm{m}^{2}$ and $8.4(3 * 0.56)$ $\mathrm{m} 2$; All the windows are of casement types without any blinds or shading devices; The area of the door on the east side is 2 $(2 * 1) \mathrm{m}^{2}$; All the walls and flat roofs of the house are comprised of the same structural insulated panels [31].

The discount rate is $6 \%$ and load growth rates for power and thermal demands are set as $6.7 \%$ and $5 \%$ respectively [5], [27].

TABLE III

ENERgy TARIFFS AND EMISSION PRICES [17], [19], [27]

\begin{tabular}{c|c|c|cc}
\hline \hline Type & Price & Type & $\begin{array}{c}\text { Price } \\
(\$ / \mathrm{kWh})\end{array}$ & Period(h) \\
\hline Local Power & 0.18 & Power & 0.0468 & $0-6,23-24$ \\
Local Thermal & 0.022 & Transaction & 0.1029 & $6-8,11-17$ \\
Natural Gas & 0.039 & & 0.1785 & $8-11,17-22$ \\
\hline Emission Price & CCHP & $0.0057(\$ / \mathrm{kW})$ & Utility Grid & $0.028(\$ / \mathrm{kW})$ \\
\hline \hline
\end{tabular}

Without loss of generality, any other real-world data can also be applied in the proposed without changing its effectiveness. The mathematical model is realized in General Algebraic Modelling System (GAMS) and solved by the Cplex solver.

\section{B. Investment-stage Simulation Results \& Discussion}

In the simulation, 2000 scenarios are initially generated and then reduced to 5 representative ones with the corresponding possibilities. After the simulation, the HES deployment results are shown in Tab. IV and V. The gray blocks mean that there are investment decisions at this phase and its subtraction with the corresponding size in the last phase denotes the newly installed capacity.

TABLE IV

DEPLOYMENT RESULTS FOR BATTERY STORAGE (KWH)

\begin{tabular}{ccccccccc}
\hline \hline Year/Bus & 3 & 6 & 18 & 22 & 25 & 27 & 30 & 33 \\
\hline $1-3$ & 1500 & 0 & 0 & 1500 & 1500 & 0 & 0 & 0 \\
$4-6$ & 1500 & 466.0 & 101.7 & 1500 & 1500 & 0 & 0 & 473.2 \\
$7-9$ & 1500 & 466.0 & 101.7 & 1500 & 1500 & 378.0 & 81.19 & 473.2 \\
\hline \hline \multicolumn{8}{c}{ TABLE V } \\
\multicolumn{10}{c}{ DEPLOYMENT RESULTS FOR THERMAL STORAGE (KWH) } \\
\hline \hline \multicolumn{1}{c}{ Year 1-9 } & Group 1 & Group2 & Group3 \\
\hline Cooling storage tank & 0 & & 0 & 0 \\
Heat storage tank & 1800 & & 1800 & 1800 \\
\hline \hline
\end{tabular}

From the HES deployment results, it can be seen that:

1) There is a widespread investment of BS in the RMEMG, and most capacities are installed on the candidate buses with RES, i.e., Bus $3,6,22,25$, etc. This is because, firstly, the sufficient BS capacity installed on those buses could contribute to the risk reduction rising from the stochastic power outputs of RES-based generators. Secondly, as the RES are intermittent, the investment of BS on those buses could guarantee enough power supply and avoid voltage violations. Further, in the system operation, BS can also shift energy from the valley-price periods to peak-price ones to gain profits. This function is mainly taken by the BS on bus $18,27,30,33$, but on those buses, the dispatchable generators can play the role for the voltage regulation, there is not so much BS investment.

2) There is no installation of the cooling storage tank but a full installation of the heat storage tank from the first investment phase in each thermal group. This is because to avoid the risk from the uncertainty sources and remain enough capacity after the internal leakage loss, the system chooses to install sufficient capacity of the energy storage, i.e., BS and heat storage. However, the cooling storage tank works only in summer but the heat storage tank contributes to supplying heat both in transition seasons as well as winter days. In this regard, given the larger amount of heat demands, more utilization rate but the same unit installation cost with the cooling storage tank, the invested capacity of the heat storage tank is much larger and more widespread than the cooling one.

3) The investment phase selection plays a necessary role in the HES deployment scheme. For example, on bus 6, 18, 27 and 33 , BS is installed at different phases. This contributes to avoiding any capacity redundancy at the early stage of the project, and thus enables a more economical and flexible HES deployment.

\section{Operation-stage Simulation Results \& Discussions}

To verify the effectiveness of the proposed method in the operation stage, the system dispatch results in the year 2026 are shown in Fig.8:



(a) Power balance in transition seasons



(c) Power balance condition in summer



(d) Cooling balance condition in summer 



(f) Heat balance in winter

Fig. 8 Energy balance condition in the year 2026.

It can be seen that all the units are coordinated to supply the multi-energy demands to maximize the EDP and reduce the risk at the same time: when the transaction prices are low, the thermal energy is transferred by electricity via EBs or ECs instead of the CCHP plants, to save the total fuel cost; when the transaction prices are high, the system thermal energy is served by the CCHP plants, and then the corresponding surplus power is sold to the utility grid to gain profits. As for the HES system, the BS charges at the low-price periods and discharges at high price periods to gain more profits from utility grid; For the TS, it makes the dispatch of the CCHP plants more flexible by absorbing its surplus thermal energy when there is more power generation than required from the CCHP plants, and releasing thermal energy when not so much power is needed from CCHP plants. Besides, the DSM scheme shifts the multi-energy demands to other periods for increasing the system profits. Its function for the cost-saving is verified in the next section.

The final result for the mean-risk model is $\$ 417.9$, with the EDP is positive as $\$ 712.1$ per day. It means that this project should be implemented as it will gain profit under risk.

In terms of the solution speed, there are 1128586 continuous and 138915 discrete variables, the solution time is 11.63 hours, which is efficient enough for a 9-year project planning problem.

\section{Methods Comparison}

To fully demonstrate the advantage of the proposed method, three conventional deployment methods for energy storage are compared:

Method \#1: Separate energy storage deployment method, i.e., the energy storage is installed in the respective single-energy microgrids. The gas boilers (GBs) are utilized instead of CCHP plants, ECs and EBs for supplying the thermal demands, while the RES-based generators and transacted power from the utility grid serve the system power demands [17].

After the simulation, the results indicate that there is no TS installation and the deployment results for the BS are shown in Table. V.

TABLE V

DEPLOYMENT RESUltS FOR BATTERY OF METHOD \#1 (KW)

\begin{tabular}{ccccccc}
\hline \hline Year/Bus & 3 & 6 & 18 & 22 & 25 & 33 \\
\hline $1-3$ & 1500 & 535.4 & 0 & 1500 & 1500 & 0 \\
$4-9$ & 1500 & 535.4 & 124.3 & 1500 & 1500 & 964.6 \\
\hline \hline
\end{tabular}

From the simulation results, under the mean-risk model, the BS is largely installed in the early phases to gain more profit and avoid risk from the diverse uncertainties. Further, the units for electricity generation in the microgrid are only RES-based ones, whose outputs are intermittent and fluctuate, thus the BS is mainly installed on the buses with RES to maintain the bus voltage in a secure range. At the same time, the BS could shift energy to gain profits. For the TS investment, as the GBs can just burn natural gas to generate thermal energy flexibly in the thermal system, there is no need to install TS given that it will bring extra installation and operating cost.

Since in single-energy microgrids, most of the power energy is obtained from the utility grid with a high price, besides, the efficiency of GBs for generating thermal energy is much lower than CCHP plants, the result for the mean-risk model of Method \#1 is \$102.4 per day. The final EDP is much lower that only $\$ 203.7$ per day.

Therefore, the simulation results show that the HES deployment in multi-energy systems can gain much more profits than the single-energy ones.

Method \#2: The HES deployment without the multi-energy DSM, i.e., the system power demands, indoor room temperature, and hot water temperature are set as constant and fixed. The corresponding simulation results are shown in Table. VI and VII.

TABLE VI

DEPLOYMENT RESUltS FOR BATTERY OF METHOD \#2 (KWH)

\begin{tabular}{ccccccc}
\hline \hline Year/Bus & 3 & 6 & 22 & 25 & 27 & 33 \\
\hline $7-9$ & 1500 & 562.7 & 1500 & 1500 & 428.4 & 508.9 \\
\hline \hline
\end{tabular}

Results For Thermal StORAGE OF Method \#2 (KWH)

\begin{tabular}{cccc}
\hline \hline Year 1-9 & Group 1 & Group2 & Group3 \\
\hline Cooling storage tank & 0 & 0 & 0 \\
Heat storage tank & 1800 & 1800 & 1800 \\
\hline \hline
\end{tabular}

Compared with our proposed method, as the power demands are not flexible anymore without the DSM scheme in Method \#2, all the BS are installed at the first investment phase with lower salvage value, to shift the system power energy, reduce the risk and maintain a safe voltage range for all the buses; As the thermal demands are not as flexible when the system is operating, there is the full installation of the heat storage tank to flexbilize the overall thermal energy operation and no installation of the cooling storage tank given its low usage rate as well as lower cooling demands. In this case, the result for the mean risk model is $\$ 348.6$ and the EDP is $\$ 674.2$ per day. The results show that without the DSM, the system would suffer from lower profit and higher risk, as the multi-energy DSM could provide additional flexibility for the system operation by actively responding to the energy prices and load conditions.

In this regard, involving the multi-energy DSM in the HES deployment is effective in increasing system profits and reduce the risk.

Method \#3: Deterministic HES deployment method, i.e., the forecasting of the outputs from RES-based generators, outdoor temperature, and residential hot water needs are assumed as accurate. Then after the simulation, the HES deployment results are shown in Table.VIII and IX.

TABLE VIII 
DEPLOYMENT RESUlTS FOR BATTERY OF METHOD \#3 (KWH)

\begin{tabular}{cccccccc}
\hline \hline Year/Bus & 3 & 6 & 18 & 22 & 25 & 27 & 33 \\
\hline $1-3$ & 1500 & 0 & 0 & 1500 & 678.4 & 0 & 0 \\
$4-6$ & 1500 & 428.6 & 0 & 1500 & 1137.2 & 0 & 487.5 \\
$7-9$ & 1500 & 578.3 & 179.3 & 1500 & 1500 & 254.8 & 487.5 \\
\hline \hline
\end{tabular}

Results For THERMAL STORAGE OF METHOD \#3 (KWH)

\begin{tabular}{cccc}
\hline \hline Year 1-9 & Group 1 & Group2 & Group3 \\
\hline Cooling storage tank & 0 & 0 & 0 \\
Heat storage tank & 1800 & 1800 & 1800 \\
\hline \hline
\end{tabular}

From the results, without considering the system uncertainties, i.e., there is no system operating risk, the BS is stilled largely installed at different phases to shift the power for more profits and maintain the system voltage stability; though there are the same TS deployment decisions, which play the same function as our method, the final EDP for Method \#3 is $\$ 729.7$ per day, which is higher than our method. This is because considering the diverse uncertainties will result in a large amount of risk. Hence, the corresponding EDP is lower. The cost difference between the proposed method and the deterministic approach is called Value of Perfect Information, which is used to immune against the system uncertainties as well as the potential risk.

However, to check the performance of the deterministic approach when the system uncertainties are realized, a set of 1000 random scenarios of typical days every year are utilized. The average EDP of Method \#3 is \$682.6 per day, while our method is $\$ 701.3$ per day. It means that, when the system is practically exposed to the uncertainty sources, the proposed adaptive SO method has a higher potential in gaining more profits than the deterministic one. This is because it already immunizes against the diverse uncertainties through the risk-averse stochastic process.

The results show that the proposed method can guarantee a more robust deployment plan.

\section{CONCLUSION}

This paper presents a risk-averse multi-stage stochastic HES deployment method in a RMEMG considering multi-energy DSM as well as diverse uncertainties from RES-based generators, outdoor temperature, power loads and hot-water needs. The deployment scheme is decomposed into three investment phases with the "mean-risk" as the objective. By using the scenario-based multi-stage adaptive SO method, the diverse uncertainties are handled. The case study shows that the proposed risk-averse HES deployment can help gain profits while avoiding the risk effectively. Thus, it should be implemented. Further, the comparisons with the three conventional deployment methods are done. The comparison results indicate that the system expected profit is around 3 times the separate energy storage deployment method. This means that deploying the HES in the multi-energy scheme is definitely necessary as the CCHP plants are highly efficient; Compared with the HES deployment approach without the multi-energy DSM, there is a rate of increment $5.62 \%$ in the system profit. This demonstrates the effectiveness of the DSM in the HES deployment; Further, compared with the deterministic HES deployment method, though there is a $2.47 \%$ rate of decrement in the expected profit, however, when the system is exposed to the real-life uncertainties, there is a rate of increment of $2.74 \%$ in the profit. This shows that the proposed method is effective in handling the uncertainties and avoid the risk to the system.

For the practical application, with the increasing penetration level of renewables and widespread installation of smart meters, this work could provide beneficial references for deploying the electric and thermal energy storage in the emerging smart homes, green buildings, etc., which have signed long-term contracts for the multi-energy DSM.

In the future, on the one hand, to conduct more comprehensive operation research for energy storage, the degradation model [34], [35] can be incorporated. On the other hand, as the solution dimension for the multi-energy planning and operation scheme is usually high, more advanced algorithms can be explored to improve the solution efficiency.

\section{APPENDIX}

\section{A. Model Linearization}

In this paper, the nonlinear equations are linearized as:

1) Linearization of the power demand under the price-based demand response: the stepwise price-elastic demand curve uses different price level rates to represent the price elasticity of power demands, with these price and demand response levels, the real and reactive power demands can be calculated/linearized as (assuming power factors are fixed) [2]:

$$
\begin{aligned}
& P_{D L}^{t}=P_{D L, P r}^{t} \cdot \sum_{j=1}^{N_{J}} \alpha^{t, j} \cdot L_{P L}^{t, j} \\
& Q_{D L}^{t}=Q_{D L, P r}^{t} \cdot \sum_{j=1}^{N_{J}} \alpha^{t, j} \cdot L_{Q L}^{t, j}
\end{aligned}
$$

2) Linearization of Maximum function, i.e., Eqs. (19), (30)-(31): separating the max function into two individual parts will do, and we take $\mathrm{A} \geq \max (\mathrm{B}, \mathrm{C})$ as an example as:

$$
\mathrm{A} \geq \mathrm{B} ; \quad \mathrm{A} \geq \mathrm{C}
$$

3) Linearization of the bilinear item, i.e., Eqs. (35)-(36): Take $\mathrm{z}=\mathrm{Ax}$ as an example in which $\mathrm{A}$ is a continuous variable and $\mathrm{x}$ is a binary variable, and $\mathrm{A}$ is bounded below by zero and above by $\mathrm{A}$ (or any $\overline{\mathrm{Big}} \mathrm{M}$ ), then it can be linearized as:

$$
z \leq \bar{A} \cdot x ; \quad z \leq A ; \quad z \geq A-(1-x) \cdot \bar{A} ; \quad z \geq 0
$$

\section{REFERENCE}

[1] P. Poonpun and W. T. Jewell, "Analysis of the cost per kilowatt-hour to store electricity," IEEE Trans. Energy. Convers., vol. 23, no. 2, pp. 529-534, Jun. 2008.

[2] C. Zhang, et al, "Robustly Coordinated Operation of A Multi-Energy Microgrid with Flexible Electric and Thermal Loads," IEEE Trans. Smart. Grid., vol. 10, no. 3, pp. 2765 - 2775, May. 2018.

[3] Chen, H. B. Gooi, et al, "Sizing of energy storage for microgrids, "IEEE Trans. Smart. Grid., vol. 3, no. 1, pp. 142-151, Mar. 2012.

[4] C. Thrampoulidis, et al, "Optimal placement of distributed energy storage in power networks," IEEE Trans. Automat. Contr., vol. 61, no. 2, pp. 416-429, Feb. 2016.

[5] Y. Yang, H. Li, A. Aichhorn, et al, "Sizing strategy of a distributed battery storage system with high penetration of photovoltaic for voltage regulation and peak load shaving," IEEE Trans. Smart. Grid., vol. 5, no. 2, pp. 982-991, Mar. 2014.

[6] G. Carpinelli, et al, "Optimal integration of distributed energy storage devices in smart grids," IEEE Trans. Smart. Grid., vol. 4, no. 2, pp. 985-995, Jun. 2013. 
[7] P. Xiong et al, "Optimal planning of storage in power systems integrated with wind power generation," IEEE Trans. Sustain. Energ., vol. 7, no. 1, pp. 232-240, Jan. 2016.

[8] A. S. Awad, T. H. El-Fouly, et al, "Optimal ESS allocation for a load management application," IEEE Trans. Power. Syst., vol. 30, no. 1, pp. 327-336, Jan. 2015.

[9] I. Miranda, N. Silva, et al, "A holistic approach to the integration of battery energy storage systems in island electric grids with high wind penetration," IEEE Trans. Sustain. Energ., vol. 7, no. 2, pp. 775-785, Apr. 2016.

[10] J. Zhang et al., "A bi-level program for the planning of an islanded microgrid including CAES," IEEE Trans. Ind. Appl., vol. 52, no. 4, pp. 2768-2777, Oct. 2016.

[11] C. Abbey and G. Joós, "A stochastic optimization approach to the rating of energy storage systems in wind-diesel isolated grids," IEEE Trans Power Syst, vol. 24, no. 1, pp. 418-426, Feb. 2009.

[12] H. Khorramdel, et al, "Optimal Battery Sizing in Microgrids Using Probabilistic Unit Commitment," IEEE Trans Ind. Informat., vol. 12, no. 2, pp. 834-843, Apr. 2016.

[13] S. Nojavan, M. Majidi, et al, "An efficient cost-reliability optimization model for optimal siting and sizing of energy storage system in a microgrid in the presence of responsible load management," Energy, vol. 139, no. 15, pp. 89-97, Nov. 2017.

[14] E. Hajipour, M. Bozorg, et al, "Stochastic capacity expansion planning of remote microgrids with wind farms and energy storage," IEEE Trans. Sustain. Energ., vol. 6, no. 2, pp. 491-498, Apr. 2015.

[15] P. Yang et al, "Joint optimization of hybrid energy storage and generation capacity with renewable energy," IEEE Trans. Smart. Grid., vol. 5, no. 4, pp. 1566-1574, Jul. 2014.

[16] M. Roustai, et al, "A scenario-based optimization of Smart Energy Hub operation in a stochastic environment using conditional-value-at-risk," Sustain. Cities. Soc., vol. 39, pp. 309-316, May. 2018.

[17] Z. Li and Y. Xu, "Optimal coordinated energy dispatch of a multi-energy microgrid in grid-connected and islanded modes," Appl. Energy, vol. 210, pp. 974-986, Jan. 2017.

[18] S. Sharma, Y. Xu, A. Verma, and B. K. Panigrahi, "Time-Coordinated Multi-Energy Management of Smart Buildings under Uncertainties," IEEE Trans. Ind. Informat., vol.15, no.8, Aug. 2019.

[19] Z. Li, et al., "Temporally-coordinated optimal operation of a multi-energy microgrid under diverse uncertainties," Appl. Energy, vol. 240, pp. 719-729, Apr. 2019.

[20] L. Guo, W. Liu, et al, "A two-stage optimal planning and design method for combined cooling, heat and power microgrid system," Energy Convers. Manag, vol. 74, pp. 433-445, Oct. 2013.

[21] T. Ma, J. Wu, L. et al, "The optimal structure planning and energy management strategies of smart multi-energy systems," Energy, vol. 160, pp. 122-141, Oct. 2018.

[22] P. Gabrielli, M. Gazzani, E. Martelli, and M. Mazzotti, “'Optimal design of multi-energy systems with seasonal storage," Appl. Energy, vol. 219, pp. 408-424, Jun. 2018.

[23] W. Gu, J. Wang, et al, "Optimal operation for integrated energy system considering thermal inertia of district heating network and buildings," Appl. Energy, vol. 199, pp. 234-246, Aug. 2017.

[24] C. Li, et al, "Flexible transmission expansion planning associated with large-scale wind farms integration considering demand response," IET Gener. Transm. Dis., vol. 9, no. 15, pp. 2276-2283, Jul. 2015.

[25] C. Zhang, et al, "Robust coordination of distributed generation and price-based demand response in microgrids," IEEE Trans. Smart. Grid., vol. 9, no. 5, pp. 4236 - 4247, Sep. 2018.

[26] B. Zeng, et al, "Integrated planning for a transition to the low-carbon distribution system with renewable energy generation and demand response," IEEE Trans Power Syst, vol. 29, no. 3, pp. 1153-1165, May. 2014.

[27] Z. Li, Y. Xu, et al, "Optimal placement of heterogeneous distributed generators in a grid-connected multi-energy microgrid under uncertainties," IET Renew. Power. Gener., vol.13, no.14, Oct. 2019.

[28] P. R. Thimmapuram, et al, "Modeling and simulation of price elasticity of demand using an agent-based model," in Proc. IEEE ISGT, Jan. 2010, pp. $1-8$.

[29] M. Tasdighi, H. Ghasemi, et al, "Residential microgrid scheduling based on smart meters data and temperature-dependent thermal load modeling," IEEE Trans. Smart. Grid., vol. 5, no. 1, pp. 349-357, Jan. 2014.

[30] W. Gu et al., "Residential CCHP microgrid with load aggregator: Operation mode, pricing strategy, and optimal dispatch," Appl. Energy, vol. 205, pp. 173-186, Nov.2017.
[31] S. Yao, et al, "Hybrid timescale dispatch hierarchy for combined heat and power system considering the thermal inertia of heat sector," IEEE Access, vol. 6, pp. 63033-63044, Oct. 2018.

[32] R. Schultz and S. Tiedemann, "Conditional value-at-risk in stochastic programs with mixed-integer recourse," Math. Program., vol. 105, no.1, pp. 365-386, Apr. 2006.

[33] "Battery Capacity; https://www.sciencedirect.com/topics/engineering/ battery capacity," 2017.

[34] A. Ahmadian, et al, "Cost-benefit analysis of V2G implementation in distribution networks considering PEVs battery degradation," IEEE Trans. Sustain. Energ., vol. 9, no.2, pp. 961-970, Apr. 2017.

[35] S. Sharma, et al, "Time-Coordinated Multi-Energy Management of Smart Buildings under Uncertainties," IEEE Trans. Ind. Informat., vol.15, no.8, Aug. 2019.

[36] J. Wang et al., "Economic benefits of integrating solar-powered heat pumps into a CHP system," IEEE Trans. on Sustain. Energ., vol. 9, no. 4, pp. 1702-1712, Otc. 2018.

[37] M. E. Baran and F. F. Wu, "Network reconfiguration in distribution systems for loss reduction and load balancing," IEEE Trans. Power. Deliver., vol. 4, no. 2, pp. 1401-1407, Apr. 1989.

[38] "Power prices for the Tianjin Grid; http://fzgg.tj.gov.cn/gzcx/syjgcx/gd/ 201307/t20130705_30044.shtml\#," 2019.

[39] A. Faruqui and S. Sergici, "Household response to dynamic pricing of electricity: a survey of 15 experiments," J. Regul. Econ., vol. 38, no. 2, pp. 193-225, Jan. 2010.



Zhengmao Li (S'16) received the B.E. degree in 2013 and the M.E. degree in 2016, both from Shandong University, Ji'nan, China, and Ph.D. degree at the School of Electrical and Electronic Engineering, Nanyang Technological University, Singapore, 2020. Currently, he is a visiting scholar at the Stevens Institute of Technology.

His research interests include renewable energy integration, microgrid and multi-energy system, and optimization techniques such as the approximate dynamic programming, robust optimization, stochastic optimization.



Yan Xu (S'10-M'13-SM'20) received the B.E. and M.E degrees from South China University of Technology, Guangzhou, China in 2008 and 2011, respectively, and the Ph.D. degree from The University of Newcastle, Australia, in 2013. He is now the Nanyang Assistant Professor at School of Electrical and Electronic Engineering, Nanyang Technological University (NTU), and a Cluster Director at Energy Research Institute@ NTU (ERI@N), Singapore. Previously, he held The University of Sydney Postdoctoral Fellowship in Australia.

His research interests include power system stability and control, microgrid, and data-analytics for smart grid applications.

Dr. Xu is an Editor for IEEE Transactions on Power Systems, IEEE Transactions on Smart Grid, IEEE Power Engineering Letters, CSEE Journal of Power and Energy Systems, and an Associate Editor for IET Generation, Transmission \& Distribution.



Xue Feng obtained her Ph.D. degree in electrical and electronic engineering from Nanyang technological university in June 2016. Her specialization is on energy storage system modeling and control for renewable energy systems and electric vehicles. During her course of Ph.D. study 
from 2011 to 2015, she was also involved in electric vehicle research with tum create. She joined land transport authority of Singapore and worked in e-mobility office from September 2015 to June 2017. her job scope in LTA involved project management and coordination for EV related projects, technical consultation for EV charging infrastructure development, formulation of new research directions and policy-making for the better introduction of EVs. Since September 2016, she has been working as an assistant professor in Singapore Institute of Technology.

Her research interests include microgrid operation and planning, integration of distributed resources, energy storage system modeling, planning and control.



Qiuwei Wu received the B.Eng. and M.Eng. in power system and its automation from Nanjing University of Science and Technology, Nanjing, China, in 2000 and 2003, respectively, and the Ph.D. in power system engineering from Nanyang Technological University, Singapore, in 2009.

$\mathrm{He}$ has been associate professor since September 2013 in the Centre of Electric Power and Energy (CEE), Department of Electrical Engineering, Technical University of Denmark (DTU), Kongens Lyngby, Denmark. $\mathrm{He}$ is an Editor of IEEE Transactions on Smart Grid and IEEE Power Engineering Letters. He is also the Deputy Editor-InChief of International Journal of Electrical Power and Energy Systems. He is an Associate Editor of Journal of Modern Power Systems and Clean Energy, and the Regional Editor for Europe of IET Renewable Power Generation. 